\section{Laser spectroscopy in development}

\author{
Sune Svanberg, Lund Institute of Technology, Sweden
}

\begin{abstract}
aser spectroscopy has developed into a very rich field of $L_{\text {scientific and technological endeavour with applications }}$ ranging from analytical spectroscopy to Bose-Einstein condensation in dilute alkali gases. It has provided completely new possibilities in fields as diverse as the study of combustion processes, atmospheric monitoring, water and vegetation surveillance, cultural heritage preservation and medical diagnostics. With the fast development of laser sources and auxiliary equipment, including computers, the applications of laser spectroscopy can become more widespread.

Optics, spectroscopy and laser applications are suitable hightech fields for the promotion of physical research in developing countries, because it is reasonably basic, it connects to real-world applications and it can be affordable. This has been observed by organisations such as the Optical Society of America (OSA), the International Commission on Optics (ICO), and the European Physical Society (EPS). The activities in the field co-ordinated by Gallieno Denardo at the Abdus Salam International Centre for Theoretical Physics (ICTP) in Trieste have been particularly notable. It helped establish the African LAM network (www.lamnetwork.org), presently co-ordinated by Ahmadou Wague, Dakar.
\end{abstract}

\section{Diode laser atomic spectroscopy}

The emergence of diode lasers useful for spectroscopy was a very important factor in establishing laser spectroscopy in less favoured regions. As an example, a small workshop was arranged in Lund in July of 1996 with participants from Cape Coast (Ghana), Dakar (Senegal), Khartoum (Sudan) and Nairobi (Tanzania) to build experimental set-ups, primarily for spectroscopy on rubidium vapour. Resonance cells with separated ${ }^{85} \mathrm{Rb}$ and ${ }^{87} \mathrm{Rb}$ isotopes were skilfully prepared by the University of Latvia, Riga. Each group built a complete set-up for diode laser absorption spectroscopy and could measure ground state hyperfine structure, the isotopic shift and Doppler broadening. Using saturation spectroscopy even Doppler-free signals could be observed, and upper-state hyperfine structure be studied. The project was financed jointly by the ICTP and the IPPS, Uppsala (director: Lennart Hasselgren). After the workshop the equipment was shipped to the different sites in Africa, and a Lund graduate student, Peter Kauranen, travelled around to the universities to help making everything functioning on site.

\section{Laser-induced fluorescence}

As a follow-up on the African diode-laser spectroscopy project an applications programme involving laser-induced fluorescence was proposed. At the Lund Institute of Technology a compact fluorosensor, based on a violet diode laser and an integrated spectrometer had been developed and successfully applied in vegetation monitoring and skin cancer diagnosis. The Lund group has a long experience in both these fields, which has relevance also to developing countries. The fact, that the hardware for these cutting-edge technology devices cost only 10,000 Euro, including a powerful lap-top computer for experiment control and data collection, made it a very realistic project. A one-month workshop, with the same title as the present article, was arranged in Lund in the spring of 2001, where two people from each of the African universities mentioned above participated, together with representatives from three further developing countries: Zimbabwe (Harare), Tunisia (Tunis) and Equador (Quito). Typically, a senior researcher and a student represented each physics department. In total six compact fluorosensors were assembled and tested by the enthusiastic participants, to be taken along home for local research. A picture from the system integration is shown in Fig. 1. The experimental work was mixed with intense lecturing on atomic and molecular spectroscopy, environmental monitoring, laser medicine and other applications. The Zimbabwean and Senegalese groups also comprised senior medical specialists (Dr Ntkomo Ndlovu, Radiation Therapy Unit, Harare and Prof. E. Malik Diop, ENT Department, Dakar) to facilitate collaboration between physicists and physicians in the fields of tissue diagnostics and photodynamic tumour therapy, in a similar way as is pursued since long at the Lund University Medical Laser Centre. The participants could attend diagnostics and treatment sessions of cancer patients, organised by Katarina Svanberg.

The fibre-optic fluorosensors are presently used in a variety of projects and further ones are being planned. Application fields are within the environmental, agricultural and medical fields. The project was financed jointly by the IPPS (Uppsala) and the Swedish Developmental Board SIDA/SAREC (project officer: Claes Kjellström).

Fig. 1: (below left) Gabriel Somesfalean, LTH (centre), provides an assisting hand to graduate student Ben Anderson (left) and Paul Buah Bassuah (right) in the integration of their Ghana fibre-optic fluorosensor.The operations are being supervised by Nejm-Eddine Jaidane, University of Tunis.

Fig. 2: (below right) Cesar Costa, Escuela Politecnica Nacional, Quito (second left) and Kenneth Kaduki, Nairobi (right) together with Lund graduate students Gabriel Somesfalean and Sara Pălsson in front of a setup for high-resolution diode-laser set-up, now transferred to Harare.
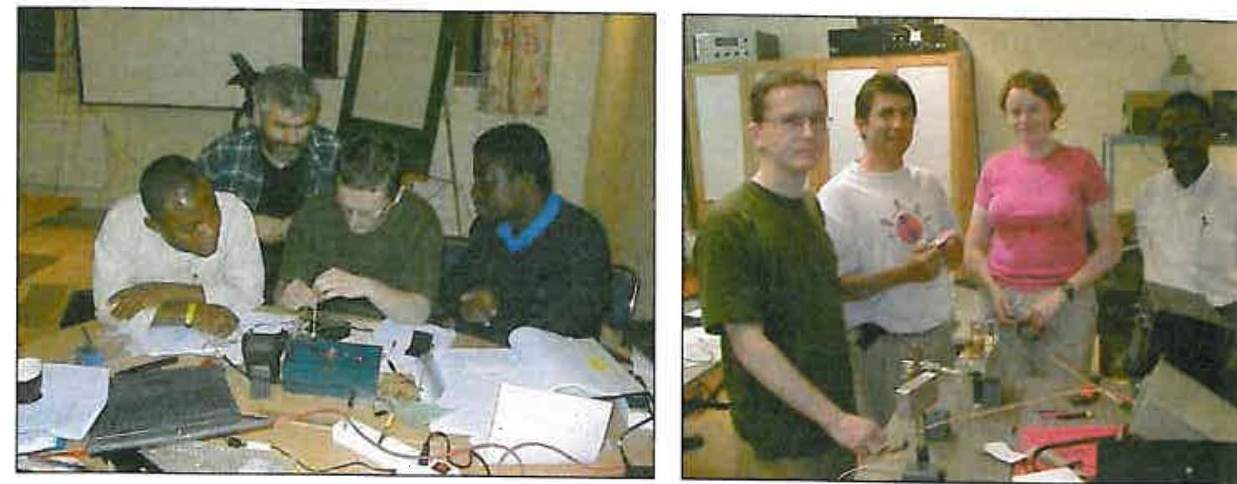


\section{Gas in scattering media absorption spectroscopy (GASMAS)}

A further technique of diode laser spectroscopy with affordable equipment and numerous applications is the newly introduced Gas in Scattering Media Absorption Spectroscopy, with the acronym GASMAS. The first paper on this technique, developed in the Lund group, had actually been published only 3 months before the 2001 workshop. A complete assembly for GASMAS was integrated during the workshop and was then shipped to Harare, within a project supported by SIDA/SAREC. A set-up for saturation spectroscopy on rubidium isotopes, similar to the ones already functioning at four other African sites, was also integrated for the Harare group and provided good training also for the all the workshop participants (See Fig. 2).

So what is the essence of the GASMAS technique? We will answer this question with reference to Fig. 3. It is a technique to study gas dispersed in scattering solids and liquids, observing that free molecules have a uniquely narrow linewidth compared to the matrix material. Normally, an extremely high resolution is not applied when studying solids or liquids, since there are no narrow features to resolve. If instead single-mode diode laser radiation is injected into the not too highly absorbing medium there will for natural materials frequently be a strong scattering and the light emerges diffusely as shown in Fig. $3 a$ and $b$. If the laser is tuned to an absorption line of, e.g. molecular oxygen, the oxygen in the pores of the material will make its narrow imprint in the emerging light. By wavelength or frequency modulation techniques, routinely applied in diode laser spectroscopy, even tiny features can be detected using the type of appartur shown in Fig. 3c. By the multiple scattering in the material the pathlength is strongly prolonged helping to increase the signal. The GASMAS technique emerged from experience obtained in Lund in the fields of diode laser spectroscopy, atmospheric laser radar, and optical mammography. The free oxygen in such diverse media as wood, marble, plastics, polystyrene, fruits and flour was successfully studied. Numerous applications in many fields, including environmental control, process steering and medicine can be envisaged using the new technique. A particularly interesting aspect is that diffusion processes can be conveniently studied: the sample is just exposed to a different atmosphere, for instance nitrogen gas, for some hours and then the time constant for the re-invasion of the atmospheric oxygen into the sample is measured! In a first example it was found that the diffusion time constant for a particular sample of polystyrene was 44 minutes. By measuring the linewidth of the signal the internal pressure in a sample can be measured! Clearly, many different gases can be assessed in different wavelength regions.

The GASMAS technique, being new, exciting and affordable (a complete set up is about 20,000 Euro) was considered to be particularly interesting for helping building research infrastructure in developing countries. Apart from the Harare facility, there are advanced plans for similar work at other locations. Presently, Benjamin Anderson from Cape Coast and Omar Marcillo from Quito are performing GASMAS experiments in Lund, together with local graduate students Mikael Sjöholm and Gabriel Somesfalean.

The three different topics introduced at the African sites high-resolution laser spectroscopy of free atoms, molecular gas monitoring in natural scattering media, and diode-laser induced fluorescence in natural samples are complementary activities. The diode laser technology is common and the laser units can be used in all three activities. While being affordable, the technology is cutting edge, and applications are realistic and also understandable to local governments. The diode laser set-ups have a

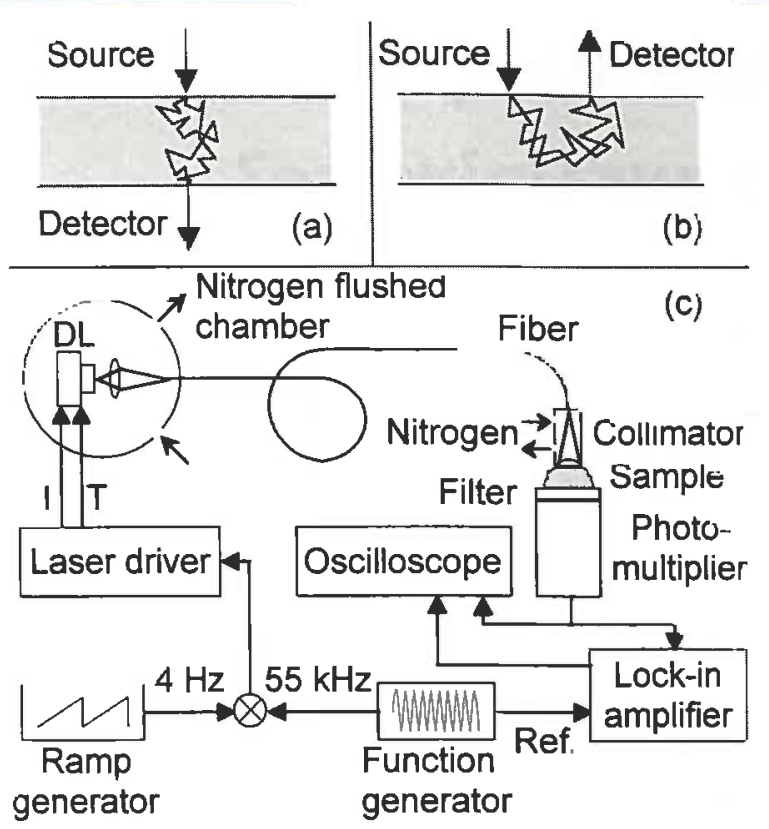

Fig. 3: Description of the GASMAS technique. In $a$ ) and $b$ ) it is shown how narrow-band diode laser radiation is injected into a porous scattering media and the emerging light is detected in two geometries.c) shows how a diode laser tuned to an molecular oxygen line around $760 \mathrm{~nm}$ is wavelength modulated to detect the sharp absorption signal in the light reaching the photomultiplier. Ambient oxygen is flushed with pure nitrogen.

wide applicability from use in lecture demonstrations and for advanced laboratory training in physics courses, to Master and $\mathrm{PhD}$ projects. With the availability of new tuneable sources such as quantum cascade lasers, a very broad wavelength region is reachable and rich possibilities for affordable further developments are evident.

\section{Bibliography}

C. Fotakis and S. Svanberg Applications of Laser Spectroscopy Europhysics News, July/August 1998 pages 151-154

S. Svanberg "Atomic and Molecular Spectroscopy - Basic Aspects and Practical Applications", Springer Verlag, Heidelberg, 3rd edn. 2001

K.B. MacAdam, A Steinbach, and C. Wieman A Narrowband Tuneable Diode Laser with Grating Feedback, and a Saturatated Absorption Spectrometer for Cs and Rb Am. J. Phys. 60 (1992) 1098

U. Gustafsson, J.Alnis, and S. Svanberg Atomic Spectroscopy with Violet Laser Diodes Am. J. Phys. 68 (2000) 660

U. Gustafsson, S. Pålsson, and S. Svanberg Compact Fibre-optic Fluorosensor using a Continuous-wave Violet Diode Laser, Rev. Sci. Instr. 71 (2000) 3004

Mikael Sjöholm, Gabriel Somesfalean, Stefan Andersson-Engels and Sune Svanberg Analysis of Gas Dispersed in Scattering Solids and Liquids, Optics Letters 26 (2001) 16 